\title{
FIXED POINTS OF NON-SMOOTH FUNCTIONS ON FINITE DIMENSIONAL ORDERED BANACH SPACES VIA CLARKE GENERALIZED JACOBIAN
}

\author{
ZOHARI AND MARDANBEIGI* \\ ${ }^{1}$ Department of mathematics, Science and Research Branch, Islamic Azad University, Tehran, Iran \\ *Corresponding author: mrmardanbeigi@srbiau.ac.ir
}

\begin{abstract}
Considering Lipschitz functions which are not necessarily Fréchet differentiable, we obtain a non-smooth version of Lakshmikantham's theorem in finite dimensional ordered Banach spaces . We also present an application of the obtained result in dynamical Coulomb friction problem.
\end{abstract}

\section{INTRODUCTION}

Ordered Banach spaces are very significant class of vector spaces which are studied widely in theory and applications of mathematics. This class of vector spaces is considered in nonlinear integral equations [2], nonlinear boundary value problems [4], optimal control theory [8], operator equations [20] and etc. On the other hand, an important theory in mathematical analysis is fixed point theory. This theory and its applications in orederd Banach spaces have been considered by many researchers. (see $[2,3,5,6,11,14,16,17]$ and the references therein.)

Recently, Lakshmikantham et al. [14] have proved some fixed point theorems in ordered Banach space $X$ for a Fréchet differentiable mapping $T: X \rightarrow X$. They showed the applications of their results in ODE initial value problems and semilinear parabolic initial boundary value problems. Vijesh and Kumar [19] and Mouhadjer and Benahmed [16] obtained some generalizations of Lakshmikantham's fixed point theorems.

Received 2019-05-21; accepted 2019-07-04; published 2019-09-02.

2010 Mathematics Subject Classification. 47H10, 49J52, 46B40.

Key words and phrases. Fixed point; Ordered Banach spaces; Clarke generalized Jacobian;Coulomb friction.

(C)2019 Authors retain the copyrights of their papers, and all open access articles are distributed under the terms of the Creative Commons Attribution License. 
To obtain fixed points in a class of nonlinear operators in ordered Banach spaces, Mouhadjer and Benahmed introduced a monotone Newton-like method in [15], by using Lakshmikantham's fixed point theorems.

In this paper, we study these fixed point theorems for Lipschitz mappings on finite Bancah spaces which are not necessary Fréchet differentiable. Our main tool is Clarke generalized Jacobian which was firstly introduced by Clarke in [9] for a mapping between two finite dimensional vector spaces. Clarke generalized Jacobian has heavy calculus rules and this paper, to the best of our knowledge, is the first work which deal to non-smooth fixed point theorem using generalized gradient. Since every finite dimensional vector space is isomorphic and homeomorphic to $\mathbb{R}^{n}$ for some $n$, we only focus on mappings $F: \mathbb{R}^{n} \rightarrow \mathbb{R}^{n}$ which are not necessary differentiable and we prove some fixed point theorems for Lipschitzian ones. Finally, to show one of the applications of the obtained results, we consider a non-smooth conic complementary problem and then we investigate the relation between obtained fixed points and the solutions of the problem. This conic complementary problem is arised in the linear discrete Coulomb friction problem which studied by Acary et al. [1].

The paper is organized as follows. In Section 2 preliminaries are given. In Section 3 we introduce some new definitions related to Clarke generalized Jacobian and then we investigate main results. Section 4 is devoted to an application of the obtained results.

\section{Preliminaries}

Let $S$ be a set. Denote the convex hull of $S$ (the set of all finite convex combinations of members of $S$ ) by $\operatorname{co}(S)$. Let $C \subseteq \mathbb{R}^{n}$ be nonempty. Then $C$ is a closed pointed convex cone, when

i) $C$ is a closed convex set,

ii) For every $x \in C$ and every scalar $\lambda \geq 0, \lambda x \in C$,

iii) $C \cap-C=\{0\}$. We have $C+C \subseteq C$ for every convex cone $C$. Using a closed pointed convex cone $C \subseteq \mathbb{R}^{n}$, we can define the following order relation on $\mathbb{R}^{n}$ :

$$
x \leq y \Longleftrightarrow y-x \in C .
$$

This relation is reflexive, antisymmetric and transitive. If $x \leq y$, then

i) $x+z \leq y+z$, for each $z \in \mathbb{R}^{n}$,

ii) $\alpha x \leq \alpha y$, for all scalar $\alpha \geq 0$.

For $\bar{x}, \bar{y} \in \mathbb{R}^{n}$ such that $\bar{x} \leq \bar{y}$, the order interval $[\bar{x}, \bar{y}]$ is defined as

$$
[\bar{x}, \bar{y}]:=\left\{z \in \mathbb{R}^{n}: \bar{x} \leq z \leq \bar{y}\right\}
$$

A cone, especially used for many applications, is standard cone in finite dimensional Euclidean spaces:

$$
\mathbb{R}_{+}^{n}:=\left\{\left(x_{1}, \ldots, x_{n}\right) \in \mathbb{R}^{n}: x_{i} \geq 0, i=1 \ldots, n\right\}
$$


Definition 2.1. Let $C \subseteq \mathbb{R}^{n}$ be a closed pointed convex cone.

i) $C$ is called normal if there exists a positive constant $\delta$ such that for every $x, y \in \mathbb{R}^{n}$,

$$
0 \leq x \leq y \Rightarrow\|x\| \leq \delta\|y\|
$$

ii) $C$ is called regular if every increasing (decreasing) sequence $\left\{x_{n}\right\}_{n \in \mathbb{N}}$ which is bounded from above (below) converges.

Since every finite-dimensional normed space is reflexive, then for closed pointed convex cone $C \subseteq \mathbb{R}^{n}$, normality and regularity are equivalent [12].

Definition 2.2. The mapping $F: \mathbb{R}^{n} \rightarrow \mathbb{R}^{m}$ is strictly differentiable at $x$, if there is an $m \times n$-matrix $M$ such that

$$
\lim _{y \rightarrow x, u \rightarrow 0} \frac{F(y+u)-F(y)-M(u)}{\|u\|}=0 .
$$

In this case, $M$ is called strict derivative of $F$ at $x$.

Definition 2.3. The mapping $F: \mathbb{R}^{n} \rightarrow \mathbb{R}^{m}$ is Fréchet differentiable at $x$, if there is an $m \times n$-matrix $M$ such that

$$
\lim _{u \rightarrow 0} \frac{F(x+u)-F(x)-M(u)}{\|u\|}=0 .
$$

In this case, $M$ is called Fréchet derivative of $F$ at $x$.

Note that, strictly differentiable functions are Fréchet differentiable while the converse is not true in general [13].

Let the vector-valued function $F: \mathbb{R}^{n} \rightarrow \mathbb{R}^{m}$ be locally Lipschitz at a given point $x$, that is, there exists a neighborhood $U$ of $x$ and a positive $k$ such that

$$
\left\|F\left(x_{1}\right)-F\left(x_{2}\right)\right\| \leq k\left\|x_{1}-x_{2}\right\|, \forall x_{1}, x_{2} \in U
$$

By Rademacher's theorem [9], $F$ is differentiable almost everywhere (in the sense of Lebesgue measure) on $U$.

Definition 2.4. $[9,13]$ Let the vector-valued function $F: \mathbb{R}^{n} \rightarrow \mathbb{R}^{m}$ be locally Lipschitz at a given point $x$. The Clarke generalized Jacobian of $F$ at $x$, denoted by $\partial F(x)$ is defined as

$$
\partial F(x):=c o\left\{\lim _{i \rightarrow \infty} \nabla F\left(x_{i}\right): x_{i} \in \Omega, x_{i} \rightarrow x\right\}
$$

where $\Omega$ is the set of points in $U$ at which $F$ is differentiable at them.

Note that $\partial F(x)$ is a nonempty convex and compact subset of $L\left(\mathbb{R}^{n}, \mathbb{R}^{m}\right)$, the space of real $m \times n$-matrices. 


\section{Main Results}

In [14], Lakshmikantham et al. considered a mapping $T: E \rightarrow E$ where $E$ is an ordered Banach space and they worked on an order interval $\left[v_{0}, w_{0}\right]$ which satisfies some assumptions. Fréchet differentiability of $T$ on $\left[v_{0}, w_{0}\right]$ is a vital assumption in [14]. One of the Lakshmikantham's abstract fixed point theorems is as follows.

Theorem 3.1. [14, Theorem 2.1] Let E be an ordered Banach space with regular order cone E $E_{+}$Suppose $T: E \rightarrow E$ satisfies the following hypotheses:

(i) There exist $v_{0}, w_{0} \in E$ such that $v_{0} \leq T v_{0}, T w_{0} \leq w_{0}$ and $v_{0} \leq w_{0}$.

(ii) The Fréchet derivative $T^{\prime}(u)$ exists for every $u \in\left[v_{0}, w_{0}\right]$, and the mapping $u \mapsto T^{\prime}(u) v$ is increasing on $\left[v_{0}, w_{0}\right]$ for all $v \in E_{+}$.

(iii) $\left[I-T^{\prime}(u)\right]^{-1}$ exists and is a bounded and positive operator for all $u \in\left[v_{0}, w_{0}\right]$.

Then, for $n \in \mathbb{N}$, relations

$$
v_{n+1}=T v_{n}+T^{\prime}\left(v_{n}\right)\left(v_{n+1}-v_{n}\right), w_{n+1}=T w_{n}+T^{\prime}\left(v_{n}\right)\left(w_{n+1}-w_{n}\right),
$$

define an increasing sequence $\left\{v_{n}\right\}_{n=0}^{\infty}$ and a decreasing sequence $\left\{w_{n}\right\}_{n=0}^{\infty}$ which both converge to fixed points of $T$. These fixed points are equal if

(iv) $T u_{1}-T u_{0}<u_{1}-u_{0}$ whenever $v_{0} \leq u_{0}<u_{1} \leq w_{0}$.

Assumptions (ii) and (iii) are deeply dependent on Fréchet differentiability of $T$ on $\left[v_{0}, w_{0}\right]$, so even for a simple function $f(x)=|x|$ on $\mathbb{R}$ we cannot use this theorem (since $f$ is not differentiable at $x=0$ ).

In this section, we assume that, the mappings are Lipschitz and we impose strictly differentiability only in $\bar{v}$, the left bound of the order interval $[\bar{v}, \bar{w}]$, and we do not need to assume differentiability in other members of the interval.

Definition 3.1. Let $F: \mathbb{R}^{n} \rightarrow \mathbb{R}^{n}$ be locally Lipschitz on $[\bar{v}, \bar{w}]$ and let $C$ be a regular convex cone. We say the set-valued $u \mapsto \partial F(u)$ is semi-increasing on $[\bar{v}, \bar{w}]$ if for all $v, w \in[\bar{v}, \bar{w}]$, with $w-v \in C$,

$$
\partial F(w) \circ z \subseteq \partial F(v) \circ z+C, \quad \forall z \in C,
$$

where o denotes the matrix multiplication.

Remark 3.1. If $F: \mathbb{R}^{n} \rightarrow \mathbb{R}^{n}$ is strictly differentiable on $[\bar{v}, \bar{w}]$, then for every $x \in[\bar{v}, \bar{w}]$, $\partial F(\cdot)$ is a singleton and Lipschitz on $[\bar{v}, \bar{w}]$ [9]. We denote this derivative by $F^{\prime}(x)$. If $F$ is semi-increasing on $[\bar{v}, \bar{w}]$ and $C=\mathbb{R}_{+}^{n}$. Then, for all $v, w \in[\bar{v}, \bar{w}]$, with $w-v \in \mathbb{R}_{+}^{n}$ we have,

$$
F^{\prime}(w) \circ z \subseteq F^{\prime}(v) \circ z+\mathbb{R}_{+}^{n}, \quad \forall z \in \mathbb{R}_{+}^{n}
$$


Thus, for some $r \in \mathbb{R}_{+}^{n}, F^{\prime}(w) \circ z=F^{\prime}(v) \circ z+r$. This shows that for $v \leq w, F^{\prime}(v) \circ z \leq F^{\prime}(w) \circ z$. Therefore, $u \mapsto T^{\prime}(u) z$ is increasing on $[\bar{v}, \bar{w}]$ for all $z \in \mathbb{R}_{+}^{n}$. Thus, semi-increasing map notion is a generalization of increasing map notion.

Example 3.1. Consider the function $F: \mathbb{R}^{2} \rightarrow \mathbb{R}^{2}$, defined by $F(x, y)=(|x|,|y|)$ on $\left[(0,0)^{T},(1,1)^{T}\right]$ and $C=\mathbb{R}_{+}^{2}$. Where $T$ denotes Matrix Transposition. Then,

$$
\begin{array}{ll}
\partial F(0,0) & =\left\{\left(\begin{array}{ll}
\alpha & 0 \\
0 & \beta
\end{array}\right): \alpha, \beta \in[-1,1]\right\} \\
\partial F(x, 0)=\left\{\left(\begin{array}{ll}
1 & 0 \\
0 & \beta
\end{array}\right): \beta \in[-1,1]\right\} & 0<x \leq 1 \\
\partial F(0, y)=\left\{\left(\begin{array}{ll}
\alpha & 0 \\
0 & 1
\end{array}\right): \alpha \in[-1,1]\right\} & 0<y \leq 1 \\
\partial F(x, y)=\left\{\left(\begin{array}{ll}
1 & 0 \\
0 & 1
\end{array}\right)\right\} & 0<x, y \leq 1 .
\end{array}
$$

If $v=(0,0)^{T}, v \leq w$ and $z=\left(z_{1}, z_{2}\right)^{T}$ is arbitrary, then Figure 1 shows that $\partial F(w) \circ z \subseteq \partial F(0,0) \circ z+\mathbb{R}_{+}^{2}$. It is easy to check that in all other cases, (3.1) holds too. Thus, $F$ is a semi-increasing map on $\left[(0,0)^{T},(1,1)^{T}\right]$.

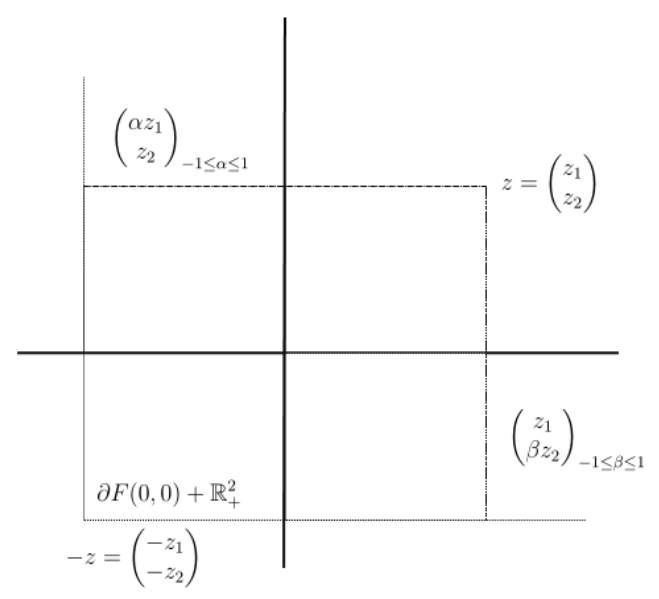

FIGURE 1. semi-increasing map in Example 3.1

Lemma 3.1. Let $F: \mathbb{R}^{n} \rightarrow \mathbb{R}^{n}$ be locally Lipschitz on $[\bar{v}, \bar{w}]$. If the set-valued $u \mapsto \partial F(u)$ is semi-increasing on $[\bar{v}, \bar{w}]$ and $v, w \in[\bar{v}, \bar{w}]$, with $w-v \in C$, then

$$
\partial F(w) \circ z \subseteq \partial F(v) \circ z-C, \quad \forall z \in-C .
$$


Proof. Let $z \in-C$ and $\zeta z \in \partial F(w) \circ z$ be arbitrary. Since $-z \in C$ and $\zeta(-z) \in \partial F(w) \circ(-z)$ we have $\zeta(-z) \in \partial F(v) \circ(-z)+C$. Thus, $\zeta(-z)=\eta(-z)+c$, for some $\eta \in \partial F(v)$ and $c \in C$. On the other hand, $\partial F(u) \subseteq L\left(\mathbb{R}^{n}\right)$ for every $u \in \mathbb{R}^{n}$, thus, $\zeta(-z)=-\zeta z$ and $\eta(-z)=-\eta z$ and we have $\zeta z=\eta z-c$. Hence, $\zeta z \in \partial F(v) \circ z-C$, which proves the lemma.

The following lemma plays an important role in the reminder of this paper.

Lemma 3.2. Let $F: \mathbb{R}^{n} \rightarrow \mathbb{R}^{n}$ be Lipschitz and semi-increasing on an order interval $[\bar{v}, \bar{w}]$. Then for $\bar{v} \leq v \leq w \leq \bar{w}$ we have,

$$
F(v)-F(w) \in \partial F(v) \circ(v-w)-C .
$$

Proof. According to the mean value theorem [9, Proposition 2.6.5],

$$
F(v)-F(w) \in \operatorname{co}(\partial F(c o\{v, w\})) \circ(v-w) .
$$

The right-hand side above denotes the convex hull of all points of the form $\eta(v-w)$, where $\eta \in \partial F(u)$ for some point $u \in c o\{v, w\}$. Assume that $u=v+t(w-v)$ where $0 \leq t \leq 1$. There exist $\lambda_{1}, \ldots, \lambda_{m} \in \mathbb{R}$ such that $\lambda_{i} \geq 0$ for $i \in\{1, \ldots, m\}$ and $\sum_{i=1}^{m} \lambda_{i}=1$ and we have,

$$
F(v)-F(w)=\left(\sum_{i=1}^{m} \lambda_{i} \zeta_{i}\right)(v-w),
$$

where $\zeta_{i} \in \partial F(u)$. On the other hand, since $v-w \in-C$ and $u-v \in C$, by Lemma 3.1 we have,

$$
\partial F(u) \circ(v-w) \subseteq \partial F(v) \circ(v-w)-C .
$$

Now, considering the convexity of $\partial F(u)$, also (3.2) and (3.3), we have

$$
F(v)-F(w) \in \partial F(v) \circ(v-w)-C .
$$

In the following, we prove our main results.

Theorem 3.2. Let $\mathbb{R}^{n}$ be ordered with regular cone $C$. Assume that the mapping $F: \mathbb{R}^{n} \rightarrow \mathbb{R}^{n}$ satisfies the following hypotheses.

(i) There exist $\bar{v}, \bar{w} \in \mathbb{R}^{n}$ with $\bar{w}-\bar{v} \in C$, such that $F$ is Lipschitz function on order interval $[\bar{v}, \bar{w}]$, $\bar{v} \leq F(\bar{v})$ and $F(\bar{w}) \leq \bar{w}$.

(ii) The set-valued $u \mapsto \partial F(u)$ is semi-increasing on $[\bar{v}, \bar{w}]$.

(iii) $F$ is strictly differentiable at $\bar{v}$ with $\partial F(\bar{v})=\left\{\zeta_{\bar{v}}\right\}$ such that $\left[I-\zeta_{\bar{v}}\right]^{-1}$ exists and is a bounded positive operator, that is, $\left[I-\zeta_{\bar{v}}\right]^{-1}(C) \subseteq C$.

Then for $n \in \mathbb{N}$, relations

$$
v_{n+1}=F\left(v_{n}\right)+\zeta_{\bar{v}}\left(v_{n+1}-v_{n}\right), w_{n+1}=F\left(w_{n}\right)+\zeta_{\bar{v}}\left(w_{n+1}-w_{n}\right),
$$


with $v_{0}:=\bar{v}$, define an increasing sequence $\left\{v_{n}\right\}_{n \in \mathbb{N}}$ and a decreasing sequence $\left\{w_{n}\right\}_{n \in \mathbb{N}}$ which both converge to fixed points of $F$. These fixed points are equal if

(iv) $F u_{1}-F u_{0}<u_{1}-u_{0}$ whenever $\bar{v} \leq u_{0}<u_{1} \leq w_{0}$.

Proof. We recall that since $F$ is strictly differentiable at $\bar{v}, \partial F(\bar{v})$ is a singleton set (see [13, P. 15]). Consider $v_{1}=F(\bar{v})+\zeta_{\bar{v}}\left(v_{1}-\bar{v}\right)$. Thus, $\left[I-\zeta_{\bar{v}}\right] v_{1}=F(\bar{v})-\zeta_{\bar{v}} \bar{v}$. This implies $v_{1}=\left[I-\zeta_{\bar{v}}\right]^{-1}\left(F(\bar{v})-\zeta_{\bar{v}} \bar{v}\right)$. Since $\left[I-\zeta_{\bar{v}}\right]^{-1}$ is bounded, $v_{1}$ is well-defined. We show that $\bar{v} \leq v_{1} \leq \bar{w}$. The assumption (1) implies:

$$
\bar{v}-v_{1} \leq F(\bar{v})-\left[F(\bar{v})+\zeta_{\bar{v}}\left(v_{1}-\bar{v}\right)\right]=\zeta_{\bar{v}}\left(\bar{v}-v_{1}\right) .
$$

Therefore, $\left[I-\zeta_{\bar{v}}\right]\left(\bar{v}-v_{1}\right) \leq 0$. Since, $\left[I-\zeta_{\bar{v}}\right]^{-1}$ is a positive operator, $\left(\bar{v}-v_{1}\right) \leq\left[I-\zeta_{\bar{v}}\right]^{-1}(0)=0$ and we have $\bar{v} \leq v_{1}$. Now, by (i)

$$
v_{1}-\bar{w} \in F(\bar{v})+\zeta_{\bar{v}}\left(v_{1}-\bar{v}\right)-F(\bar{w})-C .
$$

and using Lemma 3.2 we have,

$$
v_{1}-\bar{w} \in \partial F(\bar{v}) \circ(\bar{v}-\bar{w})+\zeta_{\bar{v}}\left(v_{1}-\bar{v}\right)-C .
$$

Therefore, for some $c \in C$ we have,

$$
v_{1}-\bar{w}=\zeta_{\bar{v}}(\bar{v}-\bar{w})+\zeta_{\bar{v}}\left(v_{1}-\bar{v}\right)-c .
$$

Then (3.5) implies:

$$
v_{1}-\bar{w} \leq \zeta_{\bar{v}}\left(v_{1}-\bar{w}\right)
$$

Thus $\left[I-\zeta_{\bar{v}}\right]\left(v_{1}-\bar{w}\right) \leq 0$ and $\left(v_{1}-\bar{w}\right) \leq\left[I-\zeta_{\bar{v}}\right]^{-1}(0)=0$ which implies $v_{1} \leq \bar{w}$. In a similar way we can show that there exists a point $w_{1}$ such that $w_{1}=F(\bar{w})+\zeta_{\bar{v}}\left(w_{1}-\bar{w}\right)$ and $\bar{v} \leq w_{1} \leq \bar{w}$. We claim that $v_{1} \leq w_{1}$. To prove this claim, note that:

$$
v_{1}-w_{1}=F(\bar{v})+\zeta_{\bar{v}}\left(v_{1}-\bar{v}\right)-\left[F(\bar{w})+\zeta_{\bar{v}}\left(w_{1}-\bar{w}\right)\right] .
$$

Thus, by Lemma 3.2 we have,

$$
v_{1}-w_{1} \in \partial F(\bar{v}) \circ(\bar{v}-\bar{w})+\zeta_{\bar{v}}\left(v_{1}-\bar{v}\right)-\zeta_{\bar{v}}\left(w_{1}-\bar{w}\right)-C,
$$

similarly, $v_{1}-w_{1} \leq \zeta_{\bar{v}}\left(v_{1}-w_{1}\right)$ and we have $v_{1} \leq w_{1}$.

Up to now, we showed that $\bar{v} \leq v_{1} \leq w_{1} \leq \bar{w}$. We claim for every $j \in \mathbb{N}, \bar{v} \leq v_{j} \leq v_{j+1} \leq w_{j+1} \leq w_{j} \leq \bar{w}$. Since $\bar{v} \leq v_{1}$ and $w_{1} \leq \bar{w}$, we only need to prove $v_{j} \leq v_{j+1} \leq w_{j}$ and $v_{j+1} \leq w_{j+1} \leq w_{j}$. We just prove $v_{j} \leq v_{j+1}$ and other inequalities are obtained similarly. By Lemma 3.2

$$
\begin{aligned}
v_{j}-v_{j+1} & =F\left(v_{j-1}\right)+\zeta_{\bar{v}}\left(v_{j}-v_{j-1}\right)-\left[F\left(v_{j}\right)+\zeta_{\bar{v}}\left(v_{j+1}-v_{j}\right)\right] \\
& \in \partial F\left(v_{j-1}\right) \circ\left(v_{j-1}-v_{j}\right)+\zeta_{\bar{v}}\left(v_{j}-v_{j-1}\right)-\zeta_{\bar{v}}\left(v_{j+1}-v_{j}\right)-C
\end{aligned}
$$


Since $v_{j-1}-\bar{v} \in C$ and $v_{j-1}-v_{j} \in-C$, by Lemma 3.1 we have,

$$
v_{j}-v_{j+1} \in \partial F(\bar{v}) \circ\left(v_{j-1}-v_{j}\right)+\zeta_{\bar{v}}\left(v_{j}-v_{j-1}\right)-\zeta_{\bar{v}}\left(v_{j+1}-v_{j}\right)-C .
$$

Therefore,

$$
v_{j}-v_{j+1} \leq \zeta_{\bar{v}}\left(v_{j}-v_{j+1}\right),
$$

so we have $v_{j}-v_{j+1} \leq\left[I-\zeta_{\bar{v}}\right]^{-1}(0)=0$ which proves our claim. We found an increasing sequence $\left\{v_{n}\right\}_{n \in \mathbb{N}}$ and a decreasing sequence $\left\{w_{n}\right\}_{n \in \mathbb{N}}$ such that

$$
\bar{v} \leq v_{1} \leq \ldots \leq v_{n} \leq w_{n} \leq \ldots \leq w_{1} \leq \bar{w} .
$$

Since $C$ is a regular cone, $\left\{v_{n}\right\}_{n \in \mathbb{N}}$ and $\left\{w_{n}\right\}_{n \in \mathbb{N}}$ are convergent. Suppose $v_{n} \rightarrow v$ and $w_{n} \rightarrow w$. We will prove $v_{n+1} \rightarrow F(v)$ which shows $v$ is a fixed point of $F$. We have

$$
v_{n+1}-F(v)=F\left(v_{n}\right)+\zeta_{\bar{v}}\left(v_{n+1}-v_{n}\right)-F(v)
$$

Since $F$ is continuous and $v_{n} \rightarrow v, v_{n+1}-v_{n} \rightarrow 0$ and $F\left(v_{n}\right)-F(v) \rightarrow 0$, thus $v_{n+1} \rightarrow F(v)$, so $F(v)=v$. Similarly one can show that $F(w)=w$.

If (iv) holds and $v<w$, then $w-v=F w-F v<w-v$ which is a contradiction. And the uniqueness of the fixed point is proved.

Note that under some conditions, the sequences in Theorem 3.2, converge quadratically as the following theorem shows.

Theorem 3.3. Let $\mathbb{R}^{n}$ be ordered with regular cone $C$. Let the mapping $F: \mathbb{R}^{n} \rightarrow \mathbb{R}^{n}$ satisfies the hypotheses of Theorem 3.2 and

$$
\left\|\zeta_{v}-\zeta_{\bar{v}}\right\| \leq L\|u-v\|, \forall \zeta_{v} \in \partial F(v)
$$

whenever $\bar{v} \leq v \leq u \leq \bar{w}$. Then, the sequences $\left\{v_{n}\right\}_{n=0}^{\infty}$ and $\left\{w_{n}\right\}_{n=0}^{\infty}$ converge quadratically to the same fixed point of $F$.

Proof. Note that, by Theorem 3.2, both the sequences $\left\{v_{n}\right\}_{n=0}^{\infty}$ and $\left\{w_{n}\right\}_{n=0}^{\infty}$ converge to the same fixed point $u$ of $F$. We prove that these sequences converge quadratically. For sequence $\left\{v_{n}\right\}_{n=0}^{\infty}$ we have,

$$
\begin{aligned}
v_{n+1}-u & =F\left(v_{n}\right)-F(u)+\zeta_{\bar{v}}\left(v_{n+1}-v_{n}\right) \\
& \subseteq \partial F\left(v_{n}\right) \circ\left(v_{n}-u\right)+\zeta_{\bar{v}}\left(v_{n+1}-v_{n}\right)-C .
\end{aligned}
$$

Thus, for some $\eta_{v_{n}} \in \partial F\left(v_{n}\right)$ and $c \in C$ we have,

$$
\begin{aligned}
v_{n+1}-u & =\zeta_{v_{n}} \circ\left(v_{n}-u\right)+\zeta_{\bar{v}}\left(v_{n+1}-v_{n}\right)-c \\
& =\zeta_{v_{n}} \circ\left(v_{n}-u\right)+\zeta_{\bar{v}}\left(v_{n+1}-u\right)-\zeta_{\bar{v}}\left(v_{n}-u\right)-c .
\end{aligned}
$$


Hence,

$$
u-v_{n+1}=\zeta_{v_{n}} \circ\left(u-v_{n}\right)-\zeta_{\bar{v}}\left(v_{n+1}-u\right)+\zeta_{\bar{v}}\left(v_{n}-u\right)+c .
$$

This implies

$$
\left[I-\zeta_{\bar{v}}\right]\left(u-v_{n+1}\right)=\left(\zeta_{v_{n}}-\zeta_{\bar{v}}\right) \circ\left(u-v_{n}\right)+c .
$$

Since, $\left[I-\zeta_{\bar{v}}\right]^{-1}$ is a positive operator, we have

$$
0 \leq u-v_{n+1} \leq\left[I-\zeta_{\bar{v}}\right]^{-1}\left(\zeta_{v_{n}}-\zeta_{\bar{v}}\right) \circ\left(u-v_{n}\right)+\bar{c}
$$

where $\bar{c}=\left[I-\zeta_{\bar{v}}\right]^{-1}(c)$. On the other hand, the cone $C$ is regular and therefore normal, thus, there exists a positive constant $\delta$ such that

$$
\begin{aligned}
\left\|u-v_{n+1}\right\| & \leq \delta\left\|\left[I-\zeta_{\bar{v}}\right]^{-1}\right\|\left\|\left(\zeta_{v_{n}}-\zeta_{\bar{v}}\right)\right\|\left\|u-v_{n}\right\|+\bar{c} \\
& \leq \delta L\left\|\left[I-\zeta_{\bar{v}}\right]^{-1}\right\|\left\|u-v_{n}\right\|^{2}+\bar{c} .
\end{aligned}
$$

For sequence $\left\{w_{n}\right\}_{n=0}^{\infty}$ the claim can be proved by a similar manner.

\section{Application in the Dynamical Coulomb Friction Problem}

The dynamical Coulomb friction problem in finite dimension with discretized time is associated to the problem of simulating the dynamics of mechanical systems which involve unilateral contact between their parts or with external objects. Acary et al. [1] presented a new formulation of this problem. They capture and treat directly the friction model as a parametric quadratic optimization problem with second-order cone constraints coupled with a fixed point equation. In this section, we just use the resulted fixed point theorems obtained in this paper, to investigate problem. Firstly, we recall some preliminaries.

Let $x \in \mathbb{R}^{d}$. The normal and tangential components of $x$ with respect to a unit vector $e \in \mathbb{R}^{d}$ are defined respectively as

$$
x_{N}:=x^{T} e \in \mathbb{R}, \quad x_{T}:=x-x_{N} e \in \mathbb{R}^{d} .
$$

For the unit vector $e \in \mathbb{R}^{d}$ and parameter $\mu \in(0,+\infty)$, the second-order cone $K_{e, \mu}$ is defined by

$$
K_{e, \mu}:=\left\{x \in \mathbb{R}^{d}:\left\|x_{T}\right\| \leq \mu x_{N}\right\}
$$

If $\mu=0$ or $\mu=+\infty$, we define

$$
K_{e, 0}:=\left\{x \in \mathbb{R}^{d}: x_{T}=0, x_{N} \geq 0\right\}, K_{e, \infty}:=\left\{x \in \mathbb{R}^{d}: x_{N} \geq 0\right\}
$$

The dual cone of the second-order cone $K_{e, \mu}$ with $\mu \in(0, \infty)$ is defined as

$$
K_{e, \mu}^{*}:=\left\{s \in \mathbb{R}^{d}: x^{T} s \geq 0, \forall x \in K_{e, \mu}\right\}=K_{e, \frac{1}{\mu}} .
$$


Also with the convention $1 / 0=\infty$ and $1 / \infty=0,\left(K_{e, 0}\right)^{*}=K_{e, \infty}$ and $\left(K_{e, \infty}\right)^{*}=K_{e, 0}$. Consider $e^{1}, \ldots, e^{n} \in$ $\mathbb{R}^{d}$ and $\mu^{1}, \ldots, \mu^{n} \in[0,+\infty]$. The associated product cone and its dual cone are respectively

$$
\begin{aligned}
L & =K_{e^{1}, \mu^{1}} \times \cdots \times K_{e^{n}, \mu^{n}} \subseteq \mathbb{R}^{n d} \\
, \quad L^{*} & =K_{e^{1}, \mu^{1}}^{*} \times \cdots \times K_{e^{n}, \mu^{n}}^{*}=K_{e^{1}, \frac{1}{\mu^{1}}} \times \cdots \times K_{e^{n}, \frac{1}{\mu^{n}}} .
\end{aligned}
$$

Set

$$
I:=\left\{i \in\{1, \cdots, n\}: \mu^{i} \neq 0\right\}, \quad n_{I}:=\operatorname{Card} I
$$

Consider the problem:

$$
\begin{gathered}
M v+f=H^{T} r \\
\tilde{u}=H v+w+E s \\
L^{*} \ni \tilde{u} \perp r \in L \\
s^{i}=\left\|\tilde{u}_{T}^{i}\right\| \text { for } i \in I
\end{gathered}
$$

with respect to the variable $(v, r, \tilde{u}, s) \in \mathbb{R}^{m} \times \mathbb{R}^{n d} \times \mathbb{R}^{n d} \times \mathbb{R}^{n_{I}}$ where $\tilde{u}:=\left(\tilde{u}^{1}, \ldots, \tilde{u}^{n}\right) \in \mathbb{R}^{n d}, r:=$ $\left(r^{1}, \ldots, r^{n}\right) \in \mathbb{R}^{n d}$. Moreover, the data of the problem are

$$
e^{i} \in \mathbb{R}^{d}, \quad \mu^{i} \in[0,+\infty], \quad M \in \mathbb{R}^{m \times m}, \quad f \in \mathbb{R}^{m}, \quad H \in \mathbb{R}^{n d \times m}, \quad w \in \mathbb{R}^{n d}, \quad E \in \mathbb{R}^{n d \times n_{I}},
$$

where matrix $M$ is definite positive and matrix $E$ is constructed by concatenating $n_{I}$ columns $E_{i} \in \mathbb{R}^{n d}$, where $E_{i}$ is itself the concatenation of $n$ vectors of $\mathbb{R}^{d}$, all zeros except for the $i$-th which is $\mu^{i} e^{i}$.

Define

$$
\begin{gathered}
\bar{C}(s):=\left\{v \in \mathbb{R}^{m}: H v+w+E s \in L^{*}\right\}, \\
v(s):=\arg \min _{v \in \bar{C}(s)}\left\{\frac{1}{2} v^{T} M v+f^{T} v\right\} \in \mathbb{R}^{m}, \\
\tilde{u}(s):=H v(s)+w+E s \in \mathbb{R}^{n d},
\end{gathered}
$$

and

$$
F(s):=\left(\left\|\tilde{u}_{T}^{i}(s)\right\|\right)_{i \in I} \in \mathbb{R}^{n_{I}}
$$

The following theorem shows the relation between fixed points of $F$ and the solutions of (4.1).

Theorem 4.1. [1, Theorem 3.3] Let $\left(v^{*}, r^{*}, \tilde{u}^{*}, s^{*}\right)$ solves the problem (4.1), then $v^{*}=v\left(s^{*}\right)$ and $F\left(s^{*}\right)=s^{*}$.

In dimension two, the inverse is also hold.

Theorem 4.2. [1, Theorem 3.7] Let the dimension $d=2$. Then $\left(v^{*}, r^{*}, \tilde{u}^{*}, s^{*}\right)$ solves the problem (4.1) if and only if $v^{*}=v\left(s^{*}\right)$ and $F\left(s^{*}\right)=s^{*}$. 
Using these theorems, we can compute a fixed point of $F$ and then check whether the computed fixed point is a solution of the problem (4.1). Note that $F$ is a non-smooth function and to compute its fixed points we need to use the non-smooth version fixed point theorem presented in this paper. In the following Examples we show the efficiency of the resulted fixed point theorem.

Example 4.1. Let $d=2, n=3, e^{1}=e^{2}=e^{3}=\left[\begin{array}{l}0 \\ 1\end{array}\right], \mu^{1}=1, \mu^{2}=0, \mu^{3}=2$ and $m=2$. Then

$$
E=\left[\begin{array}{ll}
0 & 0 \\
1 & 0 \\
0 & 0 \\
0 & 0 \\
0 & 0 \\
0 & 2
\end{array}\right] .
$$

Also, $I=\{1,3\}$ and $n_{I}=2$. For $x=\left(x_{1}, x_{2}\right)^{T}$ and $e^{i}(i=1,2,3), x_{N}=x_{2}$ and $x_{T}=\left(x_{1}, 0\right)^{T}$. Moreover,

$$
\begin{array}{lll}
K_{e^{1}, \mu^{1}}=\left\{x \in \mathbb{R}^{2}:\left|x_{1}\right| \leq x_{2}\right\}, & K_{e^{1}, \mu^{1}}^{*}=K_{e^{1}, \mu^{1}}, \\
K_{e^{2}, \mu^{2}}=\left\{x \in \mathbb{R}^{2}: x_{1}=0, x_{2} \geq 0\right\}, & K_{e^{2}, \mu^{2}}^{*}=\left\{x \in \mathbb{R}^{2}: x_{2} \geq 0\right\} \\
K_{e^{3}, \mu^{3}}=\left\{x \in \mathbb{R}^{2}:\left|x_{1}\right| \leq 2 x_{2}\right\} & K_{e^{3}, \mu^{3}}^{*}=\left\{x \in \mathbb{R}^{2}:\left|x_{1}\right| \leq \frac{1}{2} x_{2}\right\} .
\end{array}
$$

These cones and their dual cones are showed in Figure 2.
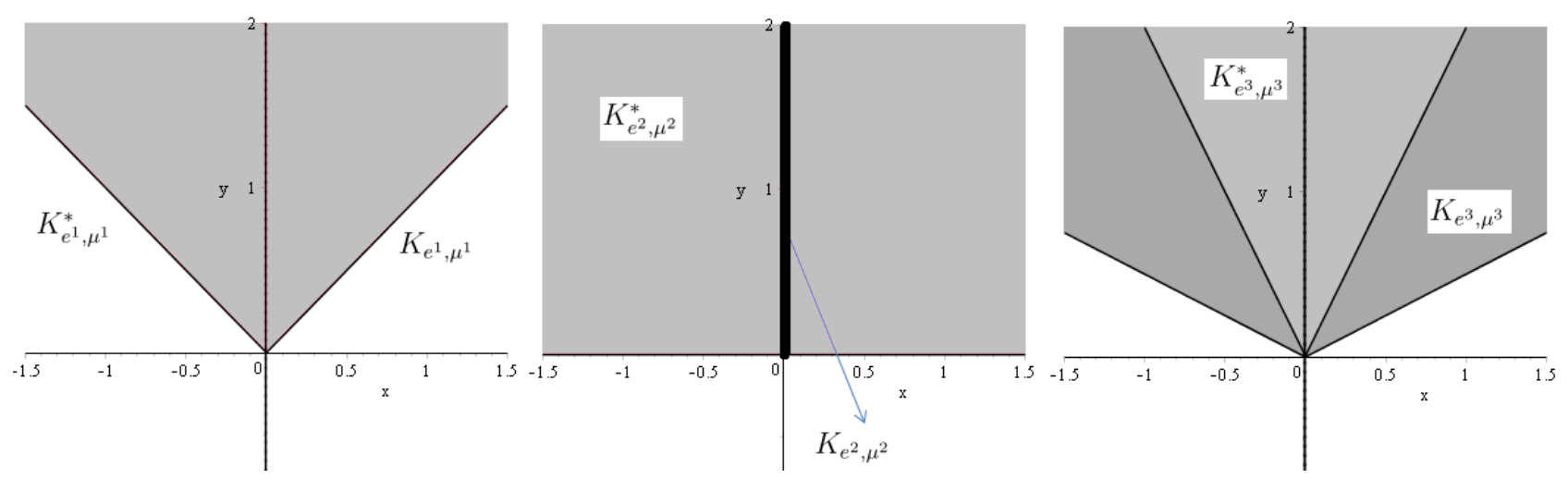

Figure 2. Cones and their dual cones in Example 4.1

Note that:

$$
\begin{aligned}
& L=\left\{(\alpha, \beta, \gamma)^{T}: \alpha \in K_{e^{1}, \mu^{1}}, \beta \in K_{e^{2}, \mu^{2}}, \gamma \in K_{e^{3}, \mu^{3}}\right\} \\
& L^{*}=\left\{\left(\alpha^{*}, \beta^{*}, \gamma^{*}\right): \alpha^{*} \in K_{e^{1}, \mu^{1}}^{*}, \beta^{*} \in K_{e^{2}, \mu^{2}}^{*}, \gamma^{*} \in K_{e^{3}, \mu^{3}}^{*}\right\}
\end{aligned}
$$


Assume,

$$
\begin{gathered}
M=\left[\begin{array}{ll}
1 & 0 \\
0 & 1
\end{array}\right], H=\left[\begin{array}{ll}
1 & 0 \\
0 & 1 \\
1 & 0 \\
1 & 0 \\
0 & 1 \\
1 & 0
\end{array}\right], w=\left[\begin{array}{l}
1 \\
0 \\
0 \\
1 \\
0 \\
0
\end{array}\right], f=\left[\begin{array}{l}
1 \\
0
\end{array}\right], v=\left[\begin{array}{l}
v_{1} \\
v_{2}
\end{array}\right], s=\left[\begin{array}{l}
s^{1} \\
s^{3}
\end{array}\right] \\
r=\left(\alpha_{1}, \alpha_{2}, \beta_{1}, \beta_{2}, \gamma_{1}, \gamma_{2}\right)^{T} \in L, \tilde{u}=\left(\alpha_{1}^{*}, \alpha_{2}^{*}, \beta_{1}^{*}, \beta_{2}^{*}, \gamma_{1}^{*}, \gamma_{2}^{*}\right)^{T} \in L^{*} .
\end{gathered}
$$

Let $v \in \bar{C}(s)$. Thus,

$$
\left[\begin{array}{l}
\alpha_{1}^{*} \\
\alpha_{2}^{*} \\
\beta_{1}^{*} \\
\beta_{2}^{*} \\
\gamma_{1}^{*} \\
\gamma_{2}^{*}
\end{array}\right] \in L^{*} \Rightarrow\left[\begin{array}{ll}
1 & 0 \\
0 & 1 \\
1 & 0 \\
1 & 0 \\
0 & 1 \\
1 & 0
\end{array}\right] \cdot\left[\begin{array}{l}
v_{1} \\
v_{2}
\end{array}\right]+\left[\begin{array}{l}
1 \\
0 \\
0 \\
1 \\
0 \\
0
\end{array}\right]+\left[\begin{array}{ll}
0 & 0 \\
1 & 0 \\
0 & 0 \\
0 & 0 \\
0 & 0 \\
0 & 2
\end{array}\right] \cdot\left[\begin{array}{l}
s^{1} \\
s^{3}
\end{array}\right] \in L^{*}
$$

Therefore,

$$
\left[\begin{array}{c}
v_{1}+1 \\
v_{2}+s^{1} \\
v_{1} \\
v_{1}+1 \\
v_{2} \\
v_{1}+2 s^{3}
\end{array}\right] \in L^{*} \Rightarrow\left[\begin{array}{c}
v_{1}+1 \\
v_{2}+s^{1}
\end{array}\right] \in K_{e^{1}, \mu^{1}}^{*},\left[\begin{array}{c}
v_{1} \\
v_{1}+1
\end{array}\right] \in K_{e^{2}, \mu^{2}}^{*},\left[\begin{array}{c}
v_{2} \\
v_{1}+2 s^{3}
\end{array}\right] \in K_{e^{3}, \mu^{3}}^{*}
$$

Hence,

$$
\left|v_{1}+1\right| \leq v_{2}+s^{1}, v_{1}+1 \geq 0,\left|v_{2}\right| \leq \frac{1}{2}\left(v_{1}+2 s^{3}\right)
$$

So, we have

$$
\bar{C}(s)=\left\{\left(v_{1}, v_{2}\right)^{T} \in \mathbb{R}^{2}: v_{1}+1 \leq v_{2}+s^{1},\left|v_{2}\right| \leq \frac{1}{2}\left(v_{1}+2 s^{3}\right)\right\} .
$$

Note that $\bar{C}(s)$ is a closed convex set and $J(v):=\frac{1}{2} v^{T} M v+f^{T} v$ is a convex function. Therefore, if $\bar{C}(s) \neq \emptyset$, then $J(v)$ has a unique solution. In this example, if $s^{1} \geq-2 s^{3}+1$, then $\bar{C}(s) \neq \emptyset$. Now we must consider the following convex optimization problem:

$$
\begin{aligned}
& \min \frac{1}{2} v^{T} M v+f^{T} v=\frac{1}{2}\left(v_{1}^{2}+v_{2}^{2}\right)+v_{1} \\
& \text { subject to } g_{1}(v):=v_{1}-v_{2}-s^{1}+1 \leq 0 \\
& g_{2}(v):=\left|v_{2}\right|-\frac{1}{2} v_{1}-s^{3} \leq 0
\end{aligned}
$$


According to [10, Page 150], if $v^{*}=\left(v_{1}^{*}, v_{2}^{*}\right)^{T}$, then there exist $\lambda_{1}, \lambda_{2} \geq 0$ such that

$$
\left(\begin{array}{l}
0 \\
0
\end{array}\right)=\left(\begin{array}{c}
v_{1}^{*}+1 \\
v_{2}^{*}
\end{array}\right)+\lambda_{1}\left(\begin{array}{c}
1 \\
-1
\end{array}\right)+\lambda_{2}\left(\begin{array}{c}
-\frac{1}{2} \\
\alpha
\end{array}\right), \lambda_{i} g_{i}\left(v^{*}\right)=0, i=1,2
$$

where

$$
\begin{cases}\alpha=1 & \text { if } v_{2}^{*}>0 \\ -1 \leq \alpha \leq 1 & \text { if } v_{2}^{*}=0 \\ \alpha=-1 & \text { if } v_{2}^{*}<0\end{cases}
$$

then $v^{*}$ is the optimal solution. The system (4.2) has a solution if $s^{3} \leq 1 / 2$. With these condition, $v_{1}^{*}=0$ and $v_{2}^{*}=-2 s^{3}$. Thus, $v(s)=\left(0,-2 s^{3}\right)$. We also have,

$$
\tilde{u}(s)=H v(s)+w+E s=\left[\begin{array}{cc}
1 & 0 \\
0 & 1 \\
1 & 0 \\
1 & 0 \\
0 & 1 \\
1 & 0
\end{array}\right]\left[\begin{array}{c}
0 \\
-2 s^{3}
\end{array}\right]+\left[\begin{array}{l}
1 \\
0 \\
0 \\
1 \\
0 \\
0
\end{array}\right]+\left[\begin{array}{ll}
0 & 0 \\
1 & 0 \\
0 & 0 \\
0 & 0 \\
0 & 0 \\
0 & 2
\end{array}\right] \cdot\left[\begin{array}{c}
s^{1} \\
s^{3}
\end{array}\right]=\left[\begin{array}{c}
1 \\
-2 s^{3}+s^{1} \\
0 \\
1 \\
-2 s^{3} \\
2 s^{3}
\end{array}\right]
$$

and

$$
F(s)=\left(1,-2 s^{3}\right), \text { where } s^{3} \leq \frac{1}{2} .
$$

Note that from Example 3.1, $F$ is a semi-increasing map with $C=\mathbb{R}_{+}^{2}$. Set $\bar{v}=(-1,-1)^{T}, \bar{w}=(2,0)^{T}$. We have $\partial F(\bar{v})=\left(\begin{array}{cc}0 & 0 \\ 0 & -2\end{array}\right)$. With these informations, conditions (i), (ii), (iii) of Theorem 3.2 hold. Set $v_{0}=(-1,-1)^{T}$ and

$$
v_{n+1}=\left(1,2\left\|v_{n}^{2}\right\|\right)^{T}+\left(\begin{array}{cc}
0 & 0 \\
0 & -2
\end{array}\right)\left(v_{n+1}-v_{n}\right) .
$$

We have $v_{n}=(1,0)^{T}$ for every $n \in \mathbb{N}$. Thus $(1,0)^{T}$ is a fixed point of $F$. It is easy to check that the combination of these resulted quantities with $r=(0,0,0,0,0,1)^{T}$, is a solution of system (4.1).

\section{References}

[1] V. Acary, F. Cadoux, C. Lemaréchal, J. Malick, A formulation of the linear discrete Coulomb friction problem via convex optimization. ZAMM, Z. Angew. Math. Mech. 91 (2011), 155-175.

[2] P.R. Agarwal, N. Hussain, M.A. Taoudi, Fixed point theorems in ordered banach spaces and applications to nonlinear integral equations, Abstr. Appl. Anal. 2012 (2012), 245872.

[3] H. Amann, Fixed point equations and nonlinear eigenvalue problems in ordered Banach spaces. SIAM rev. 18 (1976), 620-709.

[4] H. Amann, Nonlinear operators in ordered Banach spaces and some applications to nonlinear boundary value problems, In: Nonlinear Operators and the Calculus of Variations 1976, Springer-Verlag Berlin Heidelberg, pp. 1-55. 
[5] H. Andrei, P. Radu, Nonnegative solutions of nonlinear integral equations in ordered Banach spaces. Fixed Point Theory, 1 (2004), 65-70.

[6] M. Berzig, B. Samet, Positive fixed points for a class of nonlinear operatoes and applications. Positivity, 17 (2013), $235-255$.

[7] S. Bonettini, I. Loris, F. Porta, M. Prato, Variable metric inexact line-search-based methods for non-smooth optimization. SIAM J. Optim. 26 (2016), 891-921.

[8] J. Blot, N. Hayek, Infinite-Horizon Optimal Control in the Discrete-Time Framework. Springer-Verlag, New York, 2014.

[9] F.H. Clarke, Optimization and non-smooth Analysis. Society for Industrial and Applied Mathematics, 1990.

[10] A. Dhara, J. Dutta, Optimality conditions in convex optimization: a finite-dimensional view. CRC Press, 2011.

[11] T. Gnana Bhaskar, V. Lakshmikantham, Fixed point theorems in partially ordered metric spaces and applications. Nonlinear Anal. TMA, 65 (2006), 1379-1393.

[12] D. Guo, Y.J. Cho, J, Zhu, Partial ordering methods in nonlinear problems. Nova Science Publishers, New York, 2004.

[13] V. Jeyakumar, D.T. Luc, non-smooth Vector Functions and Continuous Optimization. Springer, New York, 2008.

[14] V. Lakshmikantham, S. Carl, S. Heikkilä, Fixed point theorems in ordered Banach spaces via quasilinearization. Nonlinear Anal. TMA, 71 (2009), 3448-3458.

[15] L. Mouhadjer, B. Benahmed, A Monotone Newton-Like Method for the Computation of Fixed Points, In: Le Thi H, Pham Dinh T, Nguyen N, editors. Modelling, Computation and Optimization in Information Systems and Management Sciences. Advances in Intelligent Systems and Computing, vol 359. Springer, Cham, 2015, pp. 345-356.

[16] L. Mouhadjer, B. Benahmed, Fixed point theorem in ordered Banach spaces and applications to matrix equations. Positivity, 20 (2016), 981-998.

[17] J.J. Nieto, R. Rodríguez-López, Existence and uniqueness of fixed point in partially ordered sets and applications to ordinary diferential equations. Acta Math. Sin. 3 (2007), 2203-2212.

[18] W. Rudin, Functional Analysis. McGraw-Hill, Inc. 1991.

[19] V.A. Vijesh, K.H. Kumar, Wavelet based quasilinearization method for semi-linear parabolic initial boundary value problems. Appl. Math. Comput. 266 (2015), 1163-1176.

[20] C.B. Zhai, C. Yang, C.M. Guo, Positive solutions of operator equations on ordered Banach spaces and applications. Comput. Math. Appl. 56 (2008), 3150-3156.

[21] P. Zhou, J. Du, Z. Lü, Topology optimization of freely vibrating continuum structures based on non-smooth optimization. Struct. Multidiscip. Optim. 56 (2017), 603-618. 